\title{
Estudo caso controle de alterações de creatinofosfoquinase em usuários de um laboratório do interior do Rio Grande do Sul
}

\author{
Case control study of creatine phosphokinase variation in patients \\ from a clinical laboratory of Rio Grande do Sul/Brazil
}

Danielly Joani Bulle ${ }^{1}$ Renan Vitalis', Luiza Paza', Rafaela da Silva', Clairton Edinei dos Santos', Jane Dagmar Renner ${ }^{1}$ 'Universidade de Santa Cruz do Sul (Unisc), Santa Cruz do Sul, RS, Brasil.

Recebido em: abril 2015 / Aceito em: junho 2015

daniellybulle@unisc.br

\section{RESUMO}

Objetivo: avaliar a prevalência de alterações de creatinofosfoquinase (CPK) em indivíduos em uso de sinvastatina, analisando os resultados dos exames bioquímicos de creatinofosfoquinase e relacionando com o uso da sinvastatina. Método: foi realizado um estudo caso-controle, em que foram incluídos indivíduos que fazem uso do medicamento sinvastatina (caso) e indivíduos que não utilizam sinvastatina (controle), atendidos em um laboratório ambulatorial na cidade de Santa Cruz do Sul-RS, no período de julho de 2014 a setembro de 2014. As análises foram compostas por 43 e 70 indivíduos casos e controles, respectivamente. Para ambos os grupos, foi realizada a análise dos resultados de CPK do banco de dados do laboratório em estudo. Resultados: observou-se que o aumento da enzima CPK foi significativamente maior com o uso de sinvastatina $(p<0,05)$. No grupo de casos, a prevalência de alterações musculares foi de $30,2 \%$, enquanto no grupo controle foi de $12,9 \%$. Além disso, a razão de chance de dano muscular no grupo caso é de 2,94. Considerações finais: há uma maior prevalência de alteração de CPK no grupo que utiliza sinvastatina, quando comparado com o grupo controle.

Palavras-chave: Creatinofosfoquinase; Sinvastatina; Dano muscular.

\section{ABSTRACT}

Objective: assess the prevalence of creatine phos phokinase (CPK) alterations in subjects treated with si- mvastatin. Method: we conducted a case-control study with subjects under treatment with the drug simvastatin (case group) and subjects that do not use simvastatin (control group). We included in the study subjects who underwent clinical laboratory tests in the city of Santa Cruz do Sul in from July to September 2014. Overall, we evaluated 43 subjects in the case group and 70 subjects in the control group. For both groups we performed the analysis of CPK results of laboratory database under study. Results: increase in CPK enzyme was significantly higher in the case group ( $p<0.05$ ). In this group the prevalence of muscle disorders was $30.2 \%$, and in the control group $12.9 \%$. Odds ratio of muscle damage in case group was 2.94. Closing remarks: there was a higher prevalence of CPK alterations in the group that uses simvastatin, when compared with the control group.

Keywords: Creatine Phosphokinase; Simvastatin; Muscle damage.

\section{INTRODUÇÃO}

As estatinas são medicamentos administrados por via oral, amplamente utilizados em indivíduos com hipercolesterolemia para redução dos níveis de colesterol, a fim de evitar eventos cardiovasculares. A estatina e a dose utilizada estão diretamente relacionadas com a redução do colesterol LDL (LDL-c), que pode reduzir em até $55 \%$, bem como uma elevação de colesterol HDL (HDL-c) de $5 \%$ a $10 \%$. Em indivíduos com triacilgliceróis (TG) moderadamente elevados, as estatinas podem reduzi-los em cerca de $15 \%$ a $20 \%$. Atuam 
como inibidores da HMG-CoA redutase, enzima envolvida na catálise da HMG-CoA em mevalonato-L, o qual é um intermediário chave para a biossíntese do colesterol. Com o bloqueio da via do mevalonato, mesmo que muito seguras e bem toleradas, observam-se efeitos adversos importantes como dano à musculatura esquelética. ${ }^{1-3}$

Estes fármacos podem causar uma série de efeitos adversos, incluindo particularmente o aumento transitório das transaminases e da creatinofosfoquinase (CPK). A elevação de CPK, de dez vezes ou mais, pode indicar rabdomiólise, definida como a ruptura das fibras do músculo esquelético com liberação de componentes intracelulares tóxicos para a circulação sistêmica. Muitos outros distúrbios metabólicos podem ocorrer pela lesão muscular como hipovolemia, hipercalemia, arritmia cardíaca, hipocalcemia, acidose metabólica, coagulação intravascular disseminada, falha renal aguda. Os sintomas musculares são os efeitos colaterais comuns que podem ser graves, levando a baixa adesão ou intolerância à estatina. , 4-6 $^{2,4}$

Quando ocorrem danos nos tecidos musculares, a enzima CPK, que está amplamente presente no tecido muscular, flui para a linfa, via interstício, até chegar à corrente sanguínea. A enzima CPK pode ser dosada no soro e é o exame laboratorial mais específico e sensível para avaliar dano muscular esquelético. ${ }^{7-9}$

O mecanismo da toxicidade muscular pode estar relacionado com a coenzima $\mathbf{Q} 10$, composto isoprenóide que atua na oxidação de nutrientes para produzir adenosina trifosfato (ATP). Esta etapa é importante no desempenho da função do músculo esquelético e cardíaco. Acredita-se que as estatinas causam deficiência intracelular de ubiquinona, mais especificamente bloqueando um intermediário, o farnesilpirofosfato, que é necessário para a síntese da coenzima Q10 (CoQ10). Reduzindo a CoQ10, consequentemente diminui-se a fosforilação oxidativa indispensável para o processo normal de respiração celular do miócito.,10-12

Em decorrência das alterações musculares, o objetivo deste estudo é avaliar a prevalência de alterações de creatinofosfoquinase em pacientes que frequentam um laboratório ambulatorial do interior do Rio Grande do Sul, avaliando os exames bioquímicos de CPK realizados neste local.

\section{MÉTODO}

O município de Santa Cruz do Sul fica a $150 \mathrm{Km}$ da capital Porto Alegre-RS. Possui 118.374 habitantes entre população urbana e rural. ${ }^{13} \mathrm{O}$ laboratório, onde foi realizada a pesquisa, atende a população ambulatorial do município, realizando cerca de 10.000 exames mensais e atendendo principalmente particulares e convênios.

Foi realizado um estudo caso controle em que foram incluídos 113 indivíduos acima de 40 anos, que possuíram exames bioquímicos de CPK em um laboratório de Santa Cruz do Sul (Rio Grande do Sul, Brasil), no período de julho de 2014 a setembro de 2014. A amostra foi composta por 43 casos que realizam o uso de sinvastatina de maneira contínua, e 70 controles saudáveis que não usavam sinvastatina e que foram pareados por sexo e idade. A amostra teve a proporção de uma amostra para 1,63 controle.
Foram excluídos do estudo os indivíduos que apresentavam patologias e fatores que influenciavam nas dosagens bioquímicas de CPK. O consentimento informado foi obtido de todos os pacientes e controles. $O$ estudo foi aprovado pelo Comitê de Ética da Universidade de Santa Cruz do Sul sob protocolo $n^{\circ} 726.208$, conforme a resolução do Conselho Nacional de Saúde 466/12.

Os dados clínico-epidemiológicos dos indivíduos foram obtidos na sala de espera do laboratório, através de um questionário que incluía idade, sexo, dor muscular e frequência de dor muscular. Os resultados dos exames de CPK foram obtidos do banco de dados do laboratório em estudo.

As dosagens bioquímicas foram realizadas com um equipamento da fabricante BioSystems modelo A25. Os kits utilizados foram da marca BioSystems ${ }^{\circledR}$ e a técnica das dosagens foram efetuadas conforme as instruções do fabricante. Como calibrador e controle das dosagens foram utilizados soros da mesma marca do fabricante. Os valores de CPK foram considerados normais entre 38 a $174 \mathrm{U} / \mathrm{L}$ em homens e 25 a $140 \mathrm{U} / \mathrm{L}$ em mulheres. ${ }^{14}$

Foi utilizado para as análises de dados o programa SPSS para Windows, versão 20.0 (IBM, Armonk, EUA). As associações entre as variáveis categóricas foram avaliadas pelo teste de $\chi^{2}$ ou teste exato de Fischer. A diferença entre os grupos que tiveram ou não alteração na dosagem CPK foram testadas através de análise univariada e expressa pela razão de chance (odds ratio - OR), com intervalo de confiança de 95\% (IC 95\%). Um valor de $\mathrm{p}$ menor que 0,05 foi considerado significativo.

\section{RESULTADOS}

Verificou-se que o aumento da enzima CPK foi significativamente maior com o uso de sinvastatina $(p<0,05)$ (Tabela 1). Nos casos, a prevalência de alterações musculares foi de $30,2 \%$, enquanto que no grupo controle teve prevalência de 12,9\%.

Os casos possuem uma possibilidade de desenvolverem dano muscular na razão de 13 para 30 . Este resultado indica que existe uma razão de probabilidade de 0,43 para 1 na ocorrência de dano muscular. Para pacientes que não utilizam o medicamento, a possibilidade da ocorrência de dano muscular obedece a razão 9 para 61. Isto demonstra que a razão da possibilidade de dano muscular é de 0,147 para 1.

A razão de chance de dano muscular é de 2,94 (IC $95 \%$ - descrever os valores) vezes maior de desenvolvimento de dano muscular no grupo caso, que faz tratamento com sinvastatina, em comparação ao grupo controle.

Tabela 1 - Alterações de CPK com o uso ou não de sinvastatina.

\begin{tabular}{llll}
\hline \multicolumn{4}{c}{ Sinvastatina } \\
CPK & Caso n (\%) & Controle n (\%) & $\boldsymbol{p}$ \\
\hline \multirow{4}{*}{ Normal } & $30(69,8)$ & $61(87,1)$ & \\
Aumentado & $13(30,2)$ & $9(12,9)$ & $<0,05$ \\
Total & $43(100)$ & $70(100)$ & \\
\hline
\end{tabular}

*Teste exato de Fisher. 


\section{DISCUSSÃO}

As estatinas são consideradas medicamentos relativamente seguros, raramente causam dano hepático, renal e muscular. No entanto, as reações adversas mais comuns são aquelas que afetam o músculo esquelético ocasionando mialgia, miopatia, miosite, e rabdomiólise. Os casos de miopatia são os efeitos colaterais mais comuns caracterizados por dor muscular difusa, tensão e fraqueza associada ou não a alteração dos níveis de CPK. ${ }^{15}$

A partir dos resultados obtidos, pode-se observar resultado significativo para elevação de CPK em pacientes que utilizam sinvastatina quando comparados ao grupo controle. A sinvastatina possui mecanismo de toxicidade por depleção intracelular de ubiquinona. Isto é causado pela inibição de intermediários envolvidos na síntese do colesterol que são essenciais também na síntese de ubiquinona. Esta coenzima está envolvida na oxidação de nutrientes para produção de ATP; portanto, sua diminuição intracelular resulta em diminuição da fosforilação oxidativa, necessária para o processo normal de respiração celular do miócito. ${ }^{16}$

Um estudo relatou que $81,4 \%$ das dosagens de CPK realizadas em pacientes em tratamento com sinvastatina são normais, demonstrando que existem 18,6\% de pacientes que possuem alterações musculares. Dentre os pacientes analisados neste estudo, $69,8 \%$ tiveram dosagens de CPK dentro dos valores de referência. Em uma metanálise realizada com 137.980 participantes, observou-se elevação da CPK em 778 indivíduos, não sendo significativa a prevalência de alterações musculares neste estudo; porém, as dosagens de CPK tiveram elevação quando as doses administradas de sinvastatina foram maiores, tornando-se estatisticamente significativas. ${ }^{6,17,18}$

Em um estudo realizado na Holanda pela Universidade de Groningen, Riphagen e colaboradores constataram prevalência de alterações de CPK em 8,3\% dos participantes tratados com sinvastatina, valor inferior ao demonstrado neste estudo, em que a prevalência de alterações musculares foi de 30,2\%, o que demonstra ocorrência de dano muscular frequente nos pacientes que realizam o tratamento. ${ }^{19}$

\section{CONCLUSÃO}

A partir dos dados obtidos no presente estudo, conclui-se que há uma maior prevalência de alteração de CPK no grupo que utiliza sinvastatina, quando comparado com o grupo controle.

\section{REFERÊNCIAS}

1. Itagaki M, Takaguri A, Kano S, Kaneta S, Ichihara K, Satoh $\mathrm{K}$. Possible mechanisms underlying statin-induced skeletal muscle toxicity in L6 fibroblasts and in rats. Journal of pharmacological sciences 2009; 109(1): 94-101. DOI: http:// doi.org/10.1254/jphs.08238FP.

2. Wiklund O, Pirazzi C, Romeo S. Monitoring of lipids, enzymes, and creatine kinase in patients on lipid-lowering drug therapy. Current cardiology reports 2013; 15(9): 397. DOI: http:// dx.doi.org/10.1007/s11886-013-0397-8.

3. Weber MS, Steinman L, Zamvil SS. Statins--treatment option for central nervous system autoimmune disease?
Neurotherapeutics 2007; 4(4): 693-700.

4. Kedzia A, Krysiak R, Madej A, Okopien B. [ls every case of muscle damage during hypolipemic therapy the side effect of this therapy? A case report]. Polskie Archiwum Medycyny Wewnetrznej 2007; 117(10): 473-476.

5. Tomaszewski M, Stepien KM, Tomaszewska J, Czuczwar SJ. Statin-induced myopathies. Pharmacological reports: PR. 2011; 63(4): 859-866.

6. Waness A, Bahlas S, Al Shohaib S. Simvastatin-induced rhabdomyolysis and acute renal injury. Blood purification 2008; 26(4): 394-8.

7. Silva CC, Goldberg TBL, Capela RC, Kurokawa CS, Teixeira AdS, Dalmas JC,Cyrino ES. Respostas agudas pós-exercício dos níveis de lactato sanguíneo e creatinofosfoquinase de atletas adolescentes. Rev Bras MedEsporte 2007; 13(6): 381-386. DOI: http://dx.doi.org/10.1590/S1517-86922007000600005.

8. Picarelli MM, Kaiser GRRF, Mühlen CA von. Dosagem laboratorial de enzimas musculares e diagnóstico equivocado de Polimiosite Juvenil: problemas na avaliação clínica e na fase pré-analítica. Rev Bras Reumatol 2004; 44(3): 224-226. DOI: http://dx.doi.org/10.1590/S0482-50042004000300008.

9. Pasternak RC, Smith SC, Jr., Bairey-Merz CN, Grundy SM, Cleeman Jl, Lenfant C. ACC/AHA/NHLBI clinical advisory on the use and safety of statins. J Am Coll Cardiol 2002; 106: 1024-1028. DOI: http://dx.doi.org/10.1161/01.CIR.0000032466.44170.44.

10. Jamal SM, Eisenberg MJ, Christopoulos S. Rhabdomyolysis associated with hydroxymethylglutaryl-coenzyme $A$ reductase inhibitors. American heart journal 2004; 147(6): 956-965. DOI: http://dx.doi.org/10.1016/j.ahj.2003.12.037.

11. Panchangam V. Statin-associated acute interstitial nephritis and rhabdomyolysis. Saudi journal of kidney diseases and transplantation: an official publication of the Saudi Center for Organ Transplantation, Saudi Arabia 2014; 25(3): 659-660.

12. Larsen S, Stride N, Hey-Mogensen M, Hansen $C N$, Bang LE, Bundgaard H, Nielsen LB, Helge JW, Dela F. Simvastatin effects on skeletal muscle: relation to decreased mitochondrial function and glucose intolerance. J Am Coll Cardiol 2013; 61(1): 44-53. DOI: http://dx.doi.org/10.1016/j. jacc. 2012.09.036.

13. IBGE. Instituto Brasileiro de Geografia e Estatística. 2010.

14. Motta VT. Bioquímica clínica: princípios e interpretações. Editora: Médica Massau. 2000.

15. Tomazoni SS, Leal-Junior EC, Pallotta RC, De Godoi V, Rossi RP, Frigo L, Leonardo PS, De Almeida P, Lopes-Martins, RA. Effect of simvastatin on passive strain-induced skeletal muscle injury in rats. Muscle \& nerve 2012; 46(6): 908-913. DOI: http://dx.doi.org/10.1002/mus.23444.

16. 16. Magalhães MEC. Mecanismos de rabdomiólise com as estatinas. Arq Bras de Cardiol 2005; 85(supl.5): 42-44. DOI: http://dx.doi.org/10.1590/S0066-782X2005002400011.

17. Naci H, Brugts J, Ades T. Comparative tolerability and harms of individual statins: a study-level network meta-analysis of 246955 participants from 135 randomized, controlled trials. Circulation Cardiovascular quality and outcomes 2013; 6(4): 390-9.

18. Van Staa TP, Carr DF, O'Meara H, McCann G, Pirmohamed M. Predictors and outcomes of increases in creatine phosphokinase levels or rhabdomyolysis risk during statin treatment. British journal of clinical pharmacology. 2014.

19. Riphagen IJ, van der Veer E, Muskiet FA, DeJongste MJ. Myopathy during statin therapy in the daily practice of an outpatient cardiology clinic: prevalence, predictors and relation with vitamin D. Current medical research and opinion 2012; 28(7): 1247-1452. DOI: http://dx.doi.org/10.1185/03007995. 2012.702102 . 